\title{
ON ENCLOSING SIMPLE ROOTS OF NONLINEAR EQUATIONS
}

\author{
G. ALEFELD, F. A. POTRA, AND YIXUN SHI
}

\begin{abstract}
In this paper we present two efficient algorithms for enclosing a simple root of the nonlinear equation $f(x)=0$ in the interval $[a, b]$. They improve recent methods of Alefeld and Potra by achieving higher efficiency indices and avoiding the solution of a quadratic equation per iteration. The efficiency indices of our methods are $1.5537 \ldots$ and $1.618 \ldots$, respectively. We show that our second method is an optimal algorithm in some sense. Our numerical experiments show that the two methods of the present paper compare well with the above methods of Alefeld and Potra as well as efficient solvers of Dekker, Brent, and Le. The second method in this paper has the best behavior of all, especially when the termination tolerance is small.
\end{abstract}

\section{INTRODUCTION}

In a recent paper, Alefeld and Potra [2] proposed three efficient methods for enclosing a simple zero $x_{*}$ of a continuous function $f(x)$ in the interval $[a, b]$ provided that $f(a) f(b)<0$. Starting with the initial enclosing interval $\left[a_{1}, b_{1}\right]=[a, b]$, the methods produce a sequence of intervals $\left\{\left[a_{n}, b_{n}\right]\right\}_{n=1}^{\infty}$ such that

$$
\begin{gathered}
x_{*} \in\left[a_{n+1}, b_{n+1}\right] \subseteq\left[a_{n}, b_{n}\right] \subseteq \cdots \subseteq\left[a_{1}, b_{1}\right]=[a, b], \\
\lim _{n \rightarrow \infty}\left(b_{n}-a_{n}\right)=0 .
\end{gathered}
$$

The asymptotic efficiency indices of each of those three methods, in the sense of Ostrowski [10], are $\sqrt{2}=1.4142 \ldots, \sqrt[3]{4}=1.5874 \ldots$, and $\sqrt[3]{(3+\sqrt{13}) / 2}=$ $1.4892 \ldots$, respectively. The numerical experiments in that paper show that the practical behavior of those methods is comparable to that of the efficient equation solvers of Dekker [6] and Brent [5], although they perform slightly worse on some problems.

Although there are many enclosing methods for solving the equation

$$
f(x)=0 \text {, }
$$

where $f(x)$ is continuous on $[a, b]$ and has a simple root $x_{*}$ in $[a, b]$, most of them do not have nice asymptotic convergence properties of the diameters $\left\{\left(b_{n}-a_{n}\right)\right\}_{n=1}^{\infty}$. For example, in case of Dekker's method, the diameters $b_{n}-a_{n}$ may remain greater than a relative large positive quantity until the last iteration

Received by the editor June 26, 1991 and, in revised form, June 24, 1992.

1991 Mathematics Subject Classification. Primary 65H05. 
when a " $\delta$-step" is taken. In case of Le's Algorithm LZ4 of [8], the convergence properties of $\left\{\left(b_{n}-a_{n}\right)\right\}_{n=1}^{\infty}$ have not been proved except that the total number of function evaluations will be bounded by four times that needed by the bisection method, which is also the upper bound of the number of function evaluations required by our second method in this paper. For other examples, like Brent's method, the Illinois method, the Anderson-Björck method, the Regula Falsi method, Snyder's method, the Pegasus method, and so on, only the convergence rate of $\left\{\left|x_{n}-x_{*}\right|\right\}_{n=1}^{\infty}$, where $x_{n}$ is the current estimate of $x_{*}$, has been studied and not the convergence rate of the diameters $\left(b_{n}-a_{n}\right)$.

In case $f(x)$ is convex on $[a, b]$, the classical Newton-Fourier method [10, p. 248], J. W. Schmidt's method [12], and the methods of Alefeld and Potra [1] produce a sequence of enclosing intervals whose diameters are superlinearly convergent to zero. The highest asymptotic efficiency index of those methods, $1.5537 \ldots$, is attained by a method of J. W. Schmidt [12] and a slight modification of this method due to Alefeld and Potra [1].

In the paper of Alefeld and Potra [2] three iterative methods are proposed that produce enclosing intervals satisfying (1) and (2) without any convexity assumptions on $f$. Surprisingly enough, under appropriate smoothness assumptions, one of the methods of [2] has the efficiency index $1.5874 \ldots$, which is higher than the efficiency index of the above-mentioned method of J. W. Schmidt [12].

In the present paper two new algorithms for enclosing zeros of nonconvex functions are presented. Our first method requires at most 3, while our second method requires at most 4 function evaluations per step. Both methods reduce the length of the enclosing interval by at least one half at each step, so that in the worst case scenario our methods require 3 times, respectively 4 times, more function evaluations than the bisection method. As the bisection method, or the methods of Brent [5], Dekker [6], or Le [8, 9], our methods are applicable to rather general problems involving discontinuous functions and derivatives, multiple zeros, etc. (see Theorem 3.1). However, in case of simple zeros of $C^{3}$-functions we can prove that, asymptotically, our first method requires only 2 , and our second method only 3 function evaluations per step. Moreover, in this case the sequence of diameters $\left\{\left(b_{n}-a_{n}\right)\right\}_{n=1}^{\infty}$ converges to zero with $R$ order at least $1+\sqrt{2}=2.414 \ldots$ for our first method, and $R$-order at least $2+\sqrt{5}=4.236 \ldots$ for our second method. Hence the corresponding efficiency indices are $\sqrt{1+\sqrt{2}}=1.5537 \ldots$ and $\sqrt[3]{2+\sqrt{5}}=(1+\sqrt{5}) / 2=1.618 \ldots$, respectively. As far as we know, the latter is the highest efficiency index for iterative methods that produce monotone enclosing intervals for simple zeros of sufficiently smooth functions.

This paper improves the results of [2] in two ways. First, by making better use of available information, we obtain a higher efficiency index. Second, our new algorithms do not use the exact solution of a quadratic equation at each step. Instead, we use 2 or 3 Newton steps to get a convenient approximation. This modification saves the work of computing the square root, makes the subroutine program much simpler, and preserves the good convergence properties. For convenience of comparison, we list the three algorithms of [2] in the Appendix of this paper.

In our numerical experiments we compared our methods with the methods in [2], with the methods of Dekker [6] and Brent [5] which are used in many stan- 
dard software packages, and also with the Algorithm LZ4 of Le [8]. The results are presented in $\S 5$. The numerical results show that the two methods of the present paper compare well with the other six methods. The second method in this paper has the best behavior of all, especially when the termination tolerance is small.

In $\S 6$, we show that in a certain sense our second method is an optimal procedure.

\section{Preliminary SUbroutines AND LemMas}

In this section we present some notations and results to be used later. We assume throughout that $f(x)$ is continuous on $[a, b]$ and that $f(a) f(b)<0$. We consider a point $c \in[a, b]$.

Subroutine $\operatorname{bracket}(a, b, c, \bar{a}, \bar{b}, d)$.

If $f(c)=0$, then print $c$ and stop;

If $f(a) f(c)<0$, then $\bar{a}=a, \bar{b}=c, d=b$;

If $f(b) f(c)<0$, then $\bar{a}=c, \bar{b}=b, d=a$.

After calling the above subroutine, we will have a new interval $[\bar{a}, \bar{b}] \subset[a, b]$ with $f(\bar{a}) f(\bar{b})<0$. Furthermore, we will have a point $d \notin[\bar{a}, \bar{b}]$ such that if $d<\bar{a}$ then $f(\bar{a}) f(d)>0$; otherwise $f(d) f(\bar{b})>0$.

Subroutine Newton-Quadratic $(a, b, d, r, k)$.

Set $A=f[a, b, d], B=f[a, b]$;

If $A=0$, then $r=a-B^{-1} \cdot f(a)$;

If $A \cdot f(a)>0$, then $r_{0}=a$, else $r_{0}=b$;

For $i=1,2, \ldots, k$ do:

$$
r_{i}=r_{i-1}-\frac{P\left(r_{i-1}\right)}{P^{\prime}\left(r_{i-1}\right)}=r_{i-1}-\frac{P\left(r_{i-1}\right)}{B+A\left(2 r_{i-1}-a-b\right)},
$$

$r=r_{k}$.

The above subroutine has $a, b, d$, and $k$ as inputs and $r$ as output. It is assumed that $d \notin[a, b]$ and that $f(d) f(a)>0$ if $d<a$ and $f(d) f(b)>0$ if $d>b$. Furthermore, $k$ is a positive integer and $r$ is an approximation of the unique zero $z$ of the quadratic polynomial

$$
P(x)=P(a, b, d)(x)=f(a)+f[a, b](x-a)+f[a, b, d](x-a)(x-b)
$$

in $[a, b]$, where $f[a, b]=(f(b)-f(a)) /(b-a)$, and $f[a, b, d]=$ $(f[b, d]-f[a, b]) /(d-a)$; note that, $P(a)=f(a)$ and $P(b)=f(b)$. Hence, $P(a) P(b)<0$.

Lemma 2.1. (i) Under the above assumptions, $r \in(a, b)$.

(ii) Furthermore, if $\{a, b, d\} \subseteq[e, f]$, and if $f(x)$ is twice continuously differentiable in $[e, f]$ with $f^{\prime}(x) \neq 0$ for all $x \in[e, f]$ and

$$
\delta=\min _{e \leq x \leq f}\left|f^{\prime}(x)\right|-(b-a) \max _{e \leq x \leq f}\left|f^{\prime \prime}(x)\right|>0,
$$

then

$$
|r-z| \leq \lambda^{L}(b-a)^{2^{k}}, \quad \text { where } \lambda=\frac{\max _{e \leq x \leq f\left|f^{\prime \prime}(x)\right|}}{2 \delta}, L=2^{k}-1 .
$$


Proof. (i) follows from the monotone convergence of Newton's method on quadratics, while (ii) follows by remarking that

$$
|A| \leq \frac{\max _{e \leq x \leq f}\left|f^{\prime \prime}(x)\right|}{2}
$$

and $\left|P^{\prime}(x)\right| \geq \delta>0$ for all $x \in[a, b]$. Indeed, we have that

$$
\left|r_{k}-z\right|=\left|r_{k-1}-z\right|^{2} \frac{|A|}{\left|P^{\prime}\left(r_{k-1}\right)\right|} \leq\left|r_{k-1}-z\right|^{2} \lambda \leq \lambda^{L}\left|r_{0}-z\right|^{2^{k}},
$$

where $L=1+2+\cdots+2^{k-1}=2^{k}-1$.

The next lemma can be proved in a straightforward manner; it will be needed in $\S 6$.

Lemma 2.2. Let $I_{n}=\left(n+\sqrt{1+n^{2}}\right)^{1 /(n+1)}$ for $n=1,2,3, \ldots$; then $I_{2}>I_{n}$ for all $n \neq 2$.

\section{Algorithms}

In this section we present two algorithms for enclosing a simple zero $x_{*}$ of a continuous function $f(x)$ in $[a, b]$ where $f(a) f(b)<0$. These two algorithms are improvements of the methods in [2]. The first algorithm requires at most 3 , and asymptotically 2 , function evaluations per iteration, and the second algorithm requires at most 4 , and asymptotically 3 , function evaluations per iteration. Under certain assumptions the first algorithm has an asymptotic efficiency index $\sqrt{1+\sqrt{2}}=1.5537 \ldots$ and the second algorithm has an asymptotic index $(1+\sqrt{5}) / 2=\sqrt[3]{2+\sqrt{5}}=1.6180 \ldots$ In the following algorithms, $\mu<1$ is a positive parameter which is usually chosen as $\mu=0.5$.

\section{Algorithm 1.}

1.1 set $a_{1}=a, b_{1}=b, c_{1}=a_{1}-f\left[a_{1}, b_{1}\right]^{-1} f\left(a_{1}\right)$;

1.2 call $\operatorname{bracket}\left(a_{1}, b_{1}, c_{1}, a_{2}, b_{2}, d_{2}\right)$;

For $n=2,3, \ldots$, do:

1.3 call Newton-Quadratic $\left(a_{n}, b_{n}, d_{n}, c_{n}, 2\right)$;

1.4 call $\operatorname{bracket}\left(a_{n}, b_{n}, c_{n}, \bar{a}_{n}, \bar{b}_{n}, \bar{d}_{n}\right)$;

1.5 if $\left|f\left(\bar{a}_{n}\right)\right|<\left|f\left(\bar{b}_{n}\right)\right|$, then set $u_{n}=\bar{a}_{n}$, else set $u_{n}=\bar{b}_{n}$;

1.6 set $\bar{c}_{n}=u_{n}-2 f\left[\bar{a}_{n}, \bar{b}_{n}\right]^{-1} f\left(u_{n}\right)$;

1.7 if $\left|\bar{c}_{n}-u_{n}\right|>0.5\left(\bar{b}_{n}-\bar{a}_{n}\right)$, then $\hat{c}_{n}=0.5\left(\bar{b}_{n}+\bar{a}_{n}\right)$, else $\hat{c}_{n}=\bar{c}_{n}$;

1.8 call bracket $\left(\bar{a}_{n}, \bar{b}_{n}, \hat{c}_{n}, \hat{a}_{n}, \hat{b}_{n}, \hat{d}_{n}\right)$;

1.9 if $\hat{b}_{n}-\hat{a}_{n}<\mu\left(b_{n}-a_{n}\right)$, then $a_{n+1}=\hat{a}_{n}, b_{n+1}=b_{n}, d_{n+1}=\hat{d}_{n}$, else call $\operatorname{bracket}\left(\hat{a}_{n}, \hat{b}_{n}, 0.5\left(\hat{a}_{n}+\hat{b}_{n}\right), a_{n+1}, b_{n+1}, d_{n+1}\right)$.

\section{Algorithm 2.}

2.1-2.2: same as 1.1-1.2;

For $n=2,3, \ldots$, do :

2.3 call Newton-Quadratic $\left(a_{n}, b_{n}, d_{n}, c_{n}, 2\right)$;

2.4 call $\operatorname{bracket}\left(a_{n}, b_{n}, c_{n}, \tilde{a}_{n}, \tilde{b}_{n}, \tilde{d}_{n}\right)$;

2.5 call Newton-Quadratic $\left(\tilde{a}_{n}, \tilde{b}_{n}, \tilde{d}_{n}, \tilde{c}_{n}, 3\right)$;

2.6 call $\operatorname{bracket}\left(\tilde{a}_{n}, \tilde{b}_{n}, \tilde{c}_{n}, \bar{a}_{n}, \bar{b}_{n}, \bar{d}_{n}\right)$;

2.7-2.11: same as 1.5-1.9. 
The following theorem is a basic property of the above two algorithms, whose proof is straightforward and hence will be omitted.

Theorem 3.1. Let $f$ be a real function defined on $[a, b]$ such that $f(a) f(b)<0$, and consider one of the Algorithms 1 or 2. Then either a zero of $f$ is found in a finite number of steps, or an infinite sequence of intervals $\left[a_{n}, b_{n}\right]$ is produced such that

$$
\begin{aligned}
& f\left(a_{n}\right) f\left(b_{n}\right)<1, \\
& a_{n} \leq a_{n+1} \leq b_{n+1} \leq b_{n}, \\
& b_{n+1}-a_{n+1} \leq \frac{1}{2}\left(b_{n}-a_{n}\right), \\
& \lim _{n \rightarrow \infty} a_{n}=x_{*}=\lim _{n \rightarrow \infty} b_{n}, \\
& f\left(x_{*}-0\right) f\left(x_{*}+0\right) \leq 0 .
\end{aligned}
$$

Corollary 3.2. Under the hypothesis of Theorem 3.1, assume that $f$ is continuous at $x_{*}$. Then $x_{*}$ is a zero of $f$.

\section{CONVERGENCE RESUlts}

In $\S 3$ it is easy to see that the intervals $\left\{\left[a_{n}, b_{n}\right]\right\}_{n=1}^{\infty}$ produced by either Algorithm 1 or Algorithm 2 satisfy that $b_{n+1}-a_{n+1} \leq \mu_{1}\left(b_{n}-a_{n}\right)$ for $n \geq 2$, where $\mu_{1}=\max \{\mu, 0.5\}$. Since $\mu_{1}<1$, this shows at least linear convergence. In what follows we show that under certain smoothness assumptions, Algorithm 1 and Algorithm 2 produce intervals whose diameters $\left\{\left(b_{n}-a_{n}\right)\right\}_{n=1}^{\infty}$ converge to zero with $R$-orders at least $1+\sqrt{2}=2.414 \ldots$ and $2+\sqrt{5}=4.236 \ldots$, respectively.

First, we have the following two lemmas.

Lemma 4.1 (Alefeld and Potra [2]). Assume that $f$ is continuously differentiable in $[a, b]$ and $f(a) f(b)<0$, and $x_{*}$ is a simple zero of $f(x)$ in $[a, b]$. Suppose that Algorithm 1 (or Algorithm 2) does not terminate after a finite number of iterations. Then there is an $n_{3}$ such that for all $n>n_{3}$, the quantities $\bar{c}_{n}$ and $u_{n}$ in step 1.6 (or in step 2.8) satisfy that

$$
f\left(\bar{c}_{n}\right) f\left(u_{n}\right)<0 .
$$

Lemma 4.2. Under the assumptions of Lemma 4.1, also assume that $f(x)$ is three times continuously differentiable on $[a, b]$; then

(i) for Algorithm 1, there are an $r_{1}>0$ and an $n_{1}$ such that for all $n>n_{1}$

$$
\left|f\left(c_{n}\right)\right| \leq r_{1}\left(b_{n}-a_{n}\right)^{2}\left(b_{n-1}-a_{n-1}\right),
$$

where $c_{n}$ is defined in step 1.3;

(ii) for Algorithm 2, there are an $r_{2}>0$ and an $n_{2}$ such that for all $n>n_{2}$

$$
\left|f\left(\tilde{c}_{n}\right)\right| \leq r_{2}\left(b_{n}-a_{n}\right)^{4}\left(b_{n-1}-a_{n-1}\right),
$$

where $\tilde{c}_{n}$ is defined in step 2.5.

Proof. By Theorem 3.1, $b_{n}-a_{n} \rightarrow 0$ and $x_{*} \in\left(a_{n}, b_{n}\right)$. Since $x_{*}$ is a simple root, $f^{\prime}\left(x_{*}\right) \neq 0$. Therefore, when $n$ is big enough, $f^{\prime}(x) \neq 0$ for all $x \in$ $\left[a_{n}, b_{n}\right]$. For simplicity, we assume that $f^{\prime}(x) \neq 0$ for all $x \in[a, b]$. Also, it is easy to see that in both algorithms we have that

$$
b_{n}-a_{n} \leq \mu\left(b_{n-1}-a_{n-1}\right)<\left(b_{n-1}-a_{n-1}\right) \text {. }
$$


Since $\lambda_{0}=\min _{a \leq x \leq b}\left|f^{\prime}(x)\right|>0$ and $b_{n}-a_{n} \rightarrow 0$, then for any fixed $0<\delta<\lambda_{0}$ there is an $n_{1}$ such that for all $n>n_{1}$ we have that $b_{n}-a_{n}<1$ and

$$
\delta_{n}=\lambda_{0}-\max _{a \leq x \leq b}\left|f^{\prime \prime}(x)\right|\left(b_{n}-a_{n}\right)>\delta>0 .
$$

(i) For Algorithm 1, when $n>n_{1}$, suppose $z_{n}$ is the unique zero of $P\left(a_{n}, b_{n}, d_{n}\right)(x)$ in $\left[a_{n}, b_{n}\right]$. Then using the error formula for Lagrange interpolation, we see that

$$
\begin{aligned}
\left|f\left(z_{n}\right)\right| & \leq \lambda_{1}\left|z_{n}-a_{n}\right|\left|z_{n}-b_{n}\right|\left|z_{n}-d_{n}\right| \\
& \leq 0.25 \lambda_{1}\left(b_{n}-a_{n}\right)^{2}\left(b_{n-1}-a_{n-1}\right), \quad \text { where } \lambda_{1}=\frac{1}{3 !} \max _{a \leq x \leq b}\left|f^{\prime \prime \prime}(x)\right| .
\end{aligned}
$$

By Lemma 2.1 and (10),

$$
\begin{aligned}
\mid c_{n}- & z_{n} \mid \leq\left(\frac{\max _{a \leq x \leq b}\left|f^{\prime \prime}(x)\right|}{2 \delta_{n}}\right)^{3}\left(b_{n}-a_{n}\right)^{4}<\lambda_{2}\left(b_{n}-a_{n}\right)^{4} \\
& \quad<\lambda_{2}\left(b_{n}-a_{n}\right)^{2}\left(b_{n-1}-a_{n-1}\right), \quad \text { where } \lambda_{2}=\left(\frac{\max _{a \leq x \leq b}\left|f^{\prime \prime}(x)\right|}{2 \delta}\right)^{3} .
\end{aligned}
$$

Combining (11) and (12), we have that

$$
\left|f\left(c_{n}\right)\right| \leq\left|f\left(z_{n}\right)\right|+\left(\max _{a \leq x \leq b}\left|f^{\prime}(x)\right|\right)\left|c_{n}-z_{n}\right| \leq r_{1}\left(b_{n}-a_{n}\right)^{2}\left(b_{n-1}-a_{n-1}\right),
$$

where $r_{1}=0.25 \lambda_{1}+\lambda_{2} \max _{a \leq x \leq b}\left|f^{\prime}(x)\right|$.

(ii) For Algorithm 2, when $n>n_{1}$, we have that

$$
\left|f\left(c_{n}\right)\right|<r_{1}\left(b_{n}-a_{n}\right)^{2}\left(b_{n-1}-a_{n-1}\right),
$$

where $c_{n}$ is given by 2.3. Suppose $\tilde{z}_{n}$ is the unique zero of $P\left(a_{n}, b_{n}, c_{n}\right)(x)=$ $P\left(\tilde{a}_{n}, \tilde{b}_{n}, \tilde{d}_{n}\right)(x)$ in $\left[\tilde{a}_{n}, \tilde{b}_{n}\right]$; then as in Alefeld and Potra [2], we deduce that there is an $n_{2}$ (we can choose $n_{2}>n_{1}$ ) such that for all $n>n_{2}$

$$
\left|f\left(\tilde{z}_{n}\right)\right|<\lambda_{3}\left(b_{n}-a_{n}\right)^{2}\left|f\left(c_{n}\right)\right|, \quad \text { where } \lambda_{3}=2\left(0.25 \frac{\lambda_{1}}{\lambda_{0}}\right) .
$$

Finally, similar to (12), by Lemma 2.1 and (10),

$$
\begin{aligned}
\left|\tilde{c}_{n}-\tilde{z}_{n}\right|<\lambda_{4}\left(\tilde{b}_{n}-\tilde{a}_{n}\right)^{8}< & \lambda_{4}\left(b_{n}-a_{n}\right)^{4}\left(b_{n-1}-a_{n-1}\right), \\
& \text { where } \lambda_{4}=\left(\frac{\max _{a \leq x \leq b}\left|f^{\prime \prime}(x)\right|}{2 \delta}\right)^{7} .
\end{aligned}
$$

Combining (13), (14), and (15), when $n>n_{2}>n_{1}$, we get

$$
\left|f\left(\tilde{c}_{n}\right)\right|<\left|f\left(\tilde{z}_{n}\right)\right|+\max _{a \leq x \leq b}\left|f^{\prime}(x)\right|\left|\tilde{c}_{n}-\tilde{z}_{n}\right|<r_{2}\left(b_{n}-a_{n}\right)^{4}\left(b_{n-1}-a_{n-1}\right)
$$

with $r_{2}=\lambda_{e} r_{1}+\lambda_{4} \max _{a \leq x \leq b}\left|f^{\prime}(x)\right|$.

The following two theorems show the asymptotic convergence properties of Algorithm 1 and Algorithm 2, respectively. 
Theorem 4.3. Under the assumptions of Lemma 4.2, the sequence of diameters $\left\{\left(b_{n}-a_{n}\right)\right\}_{n=1}^{\infty}$ produced by Algorithm 1 converges to zero, and there is an $L_{1}>0$ such that

$$
b_{n+1}-a_{n+1} \leq L_{1}\left(b_{n}-a_{n}\right)^{2}\left(b_{n-1}-a_{n-1}\right), \quad \forall n=2,3, \ldots .
$$

Moreover, there is an $N_{1}$ such that for all $n>N_{1}$ we have

$$
a_{n+1}=\hat{a}_{n} \text { and } b_{n+1}=\hat{b}_{n} \text {. }
$$

Hence, when $n>N_{1}$, Algorithm 1 requires only two function evaluations per iteration.

Proof. As in the proof of Lemma 4.2, we assume without loss of generality that $f^{\prime}(x) \neq 0$ for all $x \in[a, b]$. Take $N_{1}$ such that $N_{1}>\max \left\{n_{1}, n_{3}\right\}$. Then by Lemma 4.1, (7) holds for all $n>N_{1}$. For steps 1.6-1.8 of Algorithm 1 and the fact that $u_{n}, \bar{c}_{n} \in\left[\bar{a}_{n}, \bar{b}_{n}\right]$ we deduce that

$$
\hat{b}_{n}-\hat{a}_{n} \leq\left|\bar{c}_{n}-u_{n}\right|, \quad \forall n>N_{1} .
$$

From step 1.6 we also see that

$$
\left|\bar{c}_{n}-u_{n}\right|=\left|2 f\left[\bar{a}_{n}, \bar{b}_{n}\right]^{-1} f\left(u_{n}\right)\right| \leq \frac{2}{\lambda_{0}}\left|f\left(u_{n}\right)\right| .
$$

Finally, since $c_{n} \in\left\{\bar{a}_{n}, \bar{b}_{n}\right\}$, we have that $\left|f\left(u_{n}\right)\right| \leq\left|f\left(c_{n}\right)\right|$. Combining that with (18) and (19), we have

$$
\hat{b}_{n}-\hat{a}_{n} \leq \frac{2}{\lambda_{0}}\left|f\left(c_{n}\right)\right|, \quad \forall n>N_{1} .
$$

Now by Lemma 4.2, $\left|f\left(c_{n}\right)\right| \leq r_{1}\left(b_{n}-a_{n}\right)^{2}\left(b_{n-1}-a_{n-1}\right)$; hence

$$
\hat{b}_{n}-\hat{a}_{n} \leq \frac{2}{\lambda_{0}} r_{1}\left(b_{n}-a_{n}\right)^{2}\left(b_{n-1}-a_{n-1}\right), \quad \forall n>N_{1} .
$$

Since $\left\{\left(b_{n}-a_{n}\right)\right\}_{n=1}^{\infty}$ converges to zero, if $N_{1}$ is large enough, then

$$
\hat{b}_{n}-\hat{a}_{n}<\mu\left(b_{n}-a_{n}\right), \quad \forall n>N_{1} .
$$

This shows that for all $n>N_{1}$ we will have $a_{n+1}=\hat{a}_{n}$ and $b_{n+1}=\hat{b}_{n}$. By taking

$$
L_{1} \geq \max \left\{\frac{2}{\lambda_{0}} r_{1}, \frac{\left(b_{n+1}-a_{n+1}\right)}{\left(b_{n}-a_{n}\right)^{2}\left(b_{n-1}-a_{n-1}\right)}\right\}, \quad n=2,3, \ldots, N_{1},
$$

and using (21), we obtain (17).

Corollary 4.4. Under the assumptions of Theorem 4.3, $\left\{\varepsilon_{n}\right\}_{n=1}^{\infty}=\left\{\left(b_{n}-a_{n}\right)\right\}_{n=1}^{\infty}$ converges to zero with an $R$-order at least $1+\sqrt{2}=2.414 \ldots$. Since, asymptotically, Algorithm 1 requires only two function evaluations per iteration, its efficiency index is $\sqrt{1+\sqrt{2}}=1.5537 \ldots$.

Proof. By Theorem 4.3, $\left\{\varepsilon_{n}\right\}_{n=1}^{\infty}$ converges to zero and $\varepsilon_{n+1} \leq L_{1} \varepsilon_{n}^{2} \varepsilon_{n-1}$, for $n=2,3, \ldots$, and the result follows by invoking Theorem 2.1 of [11].

Theorem 4.5. Under the assumptions of Lemma 4.2, the sequence of diameters $\left\{\left(b_{n}-a_{n}\right)\right\}_{n=1}^{\infty}$ produced by Algorithm 2 converges to zero, and there is an $L_{2}>0$ such that

$$
b_{n+1}-a_{n+1} \leq L_{2}\left(b_{n}-a_{n}\right)^{4}\left(b_{n-1}-a_{n-1}\right), \quad \forall n=2,3, \ldots
$$


Moreover, there is an $N_{2}$ such that for all $n>N_{2}$ we have

$$
a_{n+1}=\hat{a}_{n} \text { and } b_{n+1}-\hat{b}_{n} .
$$

Hence, when $n>N_{2}$, Algorithm 2 requires only three function evaluations per iteration.

Proof. The proof is almost the same as that of Theorem 4.3. We assume that $f^{\prime}(x) \neq 0$ for all $x \in[a, b]$. Take $N_{2}$ such that $N_{2}>\max \left\{n_{2}, n_{3}\right\}$. Then, when $n>N_{2}$, as in the proof of Theorem 4.3, we have that

$$
\hat{b}_{n}-\hat{a}_{n} \leq \frac{2}{\lambda_{0}}\left|f\left(\tilde{c}_{n}\right)\right| .
$$

Now by Lemma $4.2,\left|f\left(\tilde{c}_{n}\right)\right| \leq r_{2}\left(b_{n}-a_{n}\right)^{4}\left(b_{n-1}-a_{n-1}\right)$. Therefore,

$$
\hat{b}_{n}-\hat{a}_{n} \leq \frac{2}{\lambda_{0}} r_{2}\left(b_{n}-a_{n}\right)^{4}\left(b_{n-1}-a_{n-1}\right), \quad \forall n>N_{2} .
$$

The rest of the proof is similar to the corresponding part of the proof of Theorem 4.3 and is omitted.

Corollary 4.6. Under the assumptions of Theorem 4.4, $\left\{\varepsilon_{n}\right\}_{n=1}^{\infty}=\left\{\left(b_{n}-a_{n}\right)\right\}_{n=1}^{\infty}$ converges to zero with an $R$-order at least $2+\sqrt{5}=4.236 \ldots$. Since asymptotically, Algorithm 2 requires only three function evaluations per iteration, its efficiency index is $\sqrt[3]{2+\sqrt{5}}=1.618 \ldots$.

\section{NUMERICAL EXPERIMENTS}

In this section we present some numerical experiments. We compared our methods with the methods in [2], with the methods of Dekker [6] and Brent [5], and also with the Algorithm LZ4 of Le [8]. In our experiments, the parameter $\mu$ in all the methods of this paper and [2] was chosen as 0.5. For Dekker's method we translated the ALGOL 60 routine Zeroin presented in [6] into Fortran; for Brent's method we simply used the Fortran routine Zero presented in the Appendix of [5], while for the Algorithm LZ4 of Le we used his Fortran code. The machine used was Encore-Multimax, and double precision was used. The test problems are listed in Table 5.1. The termination criterion was the one used by Brent [5], i.e.

$$
b-a \leq 2 \cdot \operatorname{tole}(a, b),
$$

where $[a, b]$ is the current enclosing interval, and

$$
\text { tole }(a, b)=2 \cdot|u| \cdot \underline{\text { macheps }}+\underline{\text { tol }} \text {. }
$$

Here, $u \in\{a, b\}$ is such that $|f(u)|=\min \{|f(a)|,|f(b)|\}$, macheps is the relative machine precision, which in our case is $2.2204460492504 \times 10^{-16}$, and $\underline{t o l}$ is a user-given nonnegative number.

Owing to the above termination criterion, a natural modification of the subroutine bracket was employed in our implementations of all the methods in this paper and in [2]. The modified subroutine is the following: 
Subroutine bracket $(a, b, c, \bar{a}, \bar{b})$ (or bracket $(a, b, c, \bar{a}, \bar{b}, d)$ ).

Set $\delta=\lambda \cdot \operatorname{tole}(a, b)$ for some user-given fixed $\lambda \in(0,1)$ (in our experiments we took $\lambda=0.7)$;

if $b-a \leq 4 \delta$, then set $c=(a+b) / 2$, goto 10 ;

if $c \leq a+2 \delta$, then set $c=a+2 \delta$, goto 10 ;

if $c \geq b-2 \delta$, then set $c=b-2 \delta$, goto 10 ;

10 if $f(c)=0$, then print $c$ and terminate;

if $f(a) f(c)<0$, then $\bar{a}=a, \bar{b}=c,(d=b)$;

if $f(b) f(c)<0$, then $\bar{a}=c, \bar{b}=b,(d=a)$;

calculate tole $(\bar{a}, \bar{b})$;

if $\bar{b}-\bar{a} \leq 2 \cdot \operatorname{tole}(\bar{a}, \bar{b})$, then terminate.

In our experiments we tested all the problems listed in Table 5.1 with different user-given $\underline{\mathrm{tol}}\left(\underline{\mathrm{tol}}=10^{-7}, 10^{-10}, 10^{-15}\right.$, and 0$)$. The total number of function evaluations in solving all the problems (145 cases) are listed in Table 5.2, where BR and DE stand for Brent's method and Dekker's method, respectively, and "unsolved" means a problem is not solved within 1000 iterations. From there we see that our two methods compare well with the other six methods. The second method in this paper has the best behavior of all, especially when the termination tolerance is small. This reconfirms the fact that the efficiency

TABLE 5.1. Test problems

\begin{tabular}{|c|c|c|}
\hline function $f(x)$ & {$[a, b]$} & parameter \\
\hline $\begin{array}{l}\sin x-x / 2 \\
-2 \sum_{i=1}^{20}(2 i-5)^{2} /\left(x-i^{2}\right)^{3}\end{array}$ & $\begin{array}{l}{[\pi / 2, \pi]} \\
{\left[a_{n}, b_{n}\right]} \\
a_{n}=n^{2}+10^{-9} \\
b_{n}=(n+1)^{2}-10^{-9}\end{array}$ & $n=1(1) 19$ \\
\hline$a x e^{b x}$ & {$[-9,31]$} & $\begin{array}{l}a=-40, b=-1 \\
a=-100, b=-2 \\
a=-200, b=-3\end{array}$ \\
\hline $\begin{array}{l}x^{n}-a \\
\sin x-0.5\end{array}$ & $\begin{array}{l}{[0,5]} \\
{[0.95,4.05]} \\
0,1.5]\end{array}$ & $\begin{array}{l}a=0.2,1, n=4(2) 12 \\
a=1, n=8(2) 14\end{array}$ \\
\hline $2 x e^{-n}-2 e^{-n x}+1$ & {$[0,1]$} & $n=1(1) 5,15,20$ \\
\hline$\left[1+(1-n)^{2}\right] x-(1-n x)^{2}$ & {$[0,1]$} & $n=1,2,5,10,15,20$ \\
\hline$x^{2}-(1-x)^{n}$ & {$[0,1]$} & $n=1,2,5,10,15,20$ \\
\hline$\left[1+(1-n)^{4}\right] x-(1-n x)^{4}$ & {$[0,1]$} & $n=1,2,4,5,8,15,20$ \\
\hline$e^{-n x}(x-1)+x^{n}$ & {$[0,1]$} & $n=1,5,10,15,20$ \\
\hline$(n x-1) /((n-1) x)$ & {$[0.01,1]$} & $n=2,5,15,20$ \\
\hline $\begin{cases}0 & \text { if } x=0 \\
x e^{-x^{-2}} & \text { otherwise }\end{cases}$ & {$[-1,4]$} & \\
\hline $\begin{cases}\frac{n}{20}\left(\frac{x}{1.5}+\sin x-1\right) & \text { if } x \geq 0 \\
\frac{-n}{20} & \text { otherwise }\end{cases}$ & {$\left[-10^{4}, \pi / 2\right]$} & $n=1(1) 40$ \\
\hline $\begin{cases}e-1.859 & \text { if } x>\frac{2 \times 10^{-3}}{1+n} \\
e^{(n+1) x / 2 \times 10^{3}}-1.859 & \text { if } x \in\left[0, \frac{2 \times 10^{-3}}{1+n}\right] \\
-0.859 & \text { if } x<0\end{cases}$ & {$\left[-10^{4}, 10^{-4}\right]$} & $\begin{array}{l}n=20(1) 40 \\
n=100(100) 1000\end{array}$ \\
\hline
\end{tabular}


TABLE 5.2. Total number of function evaluations in solving all the problems listed in Table 5.1

\begin{tabular}{|c|c|c|c|c|c|c|c|c|}
\hline tol & B1 & B2 & B3 & Alg. 1 & Alg. 2 & BR & $\mathrm{DE}$ & LE \\
\hline $10^{-7}$ & 3139 & 2895 & 2580 & 2800 & 2604 & 2693 & 2658 & 2643 \\
\hline $10^{-10}$ & 3447 & 2995 & 2773 & 2990 & 2708 & 2794 & $\begin{array}{c}2819 \\
1 \text { unsolved }\end{array}$ & 2808 \\
\hline $10^{-15}$ & 3672 & 3017 & 2948 & 3134 & 2746 & 2860 & $\begin{array}{c}2955 \\
1 \text { unsolved }\end{array}$ & 2971 \\
\hline 0 & 3714 & 3041 & 3007 & 3137 & 2793 & 2873 & $\begin{array}{c}2936 \\
4 \text { unsolved }\end{array}$ & $\begin{array}{l}3025 \\
3025\end{array}$ \\
\hline
\end{tabular}

TABLE 5.3. Total number of function evaluations in solving $x^{n}=0$ in $[-1,10]$ for $n=3,5,7,9,19,25$

\begin{tabular}{|c|c|c|c|c|c|c|c|c|c|}
\hline tol & B1 & B2 & B3 & Alg.1 & Alg.2 & BR & DE & LE & BIS \\
\hline $10^{-7}$ & 402 & 510 & 384 & 355 & 349 & 434 & 1340 & 185 & 174 \\
\hline $10^{-10}$ & 561 & 718 & 529 & 521 & 461 & 611 & 1987 & 237 & 234 \\
\hline $10^{-15}$ & 785 & 1034 & 721 & 757 & 746 & 867 & 2 unsolved & 377 & 325 \\
\hline 0 & 2219 & 2959 & 1793 & 2208 & 1830 & 2624 & 6 unsolved & 1680 & 921 \\
\hline
\end{tabular}

index is an asymptotic notion. In order to give an interesting example where methods having higher efficiency index are outperformed by methods with lower efficiency indices, we compare those methods with the bisection method, solving the problem

$$
x^{n}=0, \quad n=3,5,7,9,19,25
$$

with the initial interval $[a, b]=[-1,10]$. The results are listed in Table 5.3, where BIS stands for the bisection method.

\section{Discussion}

We notice that our Algorithm 2 is an optimal procedure in the following sense. It is clear that Algorithm 2 improves our Algorithm 1 by repeating 2.32.4 in 2.5-2.6. If we repeat 2.3-2.4 a total of $k$ times, then we get an algorithm of the form

\section{Algorithm 3}

3.1-3.2: same as $2.1-2.2$;

for $n=2,3, \ldots$, do

3.3 call Newton-Quadratic $\left(a_{n}, b_{n}, d_{n}, c_{n}, 2\right)$;

3.4 call $\operatorname{bracket}\left(a_{n}, b_{n}, c_{n}, a_{n}^{(1)}, b_{n}^{(1)}, d_{n}^{(1)}\right)$;

$3.2 k+1$ call Newton-Quadratic $\left(a_{n}^{(k-1)}, b_{n}^{(k-1)}, d_{n}^{(k-1)}, \tilde{c}_{n}, k+1\right)$;

$3.2 k+2$ call bracket $\left(a_{n}^{(k-1)}, b_{n}^{(k-1)}, \tilde{c}_{n}, \bar{a}_{n}, \bar{b}_{n}, \bar{d}_{n}\right)$;

$3.2 k+3-3.2 k+7$ : same as $2.7-2.11$. 
It is clear that Algorithms 1 and 2 are special cases of Algorithm 3. Furthermore, similar to Theorem 4.3 and Theorem 4.5, we see that for Algorithm 3 ,

$$
\left(b_{n+1}-a_{n+1}\right) \leq L_{k}\left(b_{n}-a_{n}\right)^{2 k}\left(b_{n-1}-a_{n-1}\right), \quad n=2,3, \ldots,
$$

for some $L_{k}>0$. Hence, Algorithm 3 has an $R$-order at least $\tau=k+\sqrt{1+k^{2}}$, which is the positive root of the equation $t^{2}-2 k t-1=0$. Since asymptotically, Algorithm 3 requires $k+1$ function evaluations per iteration, its efficiency index is $I_{k}=\left(k+\sqrt{1+k^{2}}\right)^{1 /(k+1)}$. By Lemma 2.2, $I_{k}<I_{2}$, for all $k \neq 2$. Therefore, Algorithm 2 is the optimal choice.

\section{APPENDIX}

In what follows we list the three algorithms proposed in Alefeld and Potra [2], assuming that $f(x)$ is continuous on $[a, b]$ and $f(a) f(b)<0$. For convenience, we use the names B1, B2, and B3 for the first, the second, and the third method in [2], respectively.

\section{Algorithm B1}

set $a_{1}=a, b_{1}=b$, for $n=1,2, \ldots$ do:

B1.1 $c_{n}=a_{n}-f\left[a_{n}, b_{n}\right]^{-1} f\left(a_{n}\right)$;

B1.2-B1.5: same as 1.4-1.7 in Algorithm 1 of this paper;

B1.6 call bracket $\left(\bar{a}_{n}, \bar{b}_{n}, \hat{c}_{n}, \hat{a}_{n}, \hat{b}_{n}\right)$;

B1.7 if $\hat{b}_{n}-\hat{a}_{n}<\mu\left(b_{n}-a_{n}\right)$, then set $a_{n+1}=\hat{a}_{n}, b_{n+1}=\hat{b}_{n}$; else call $\operatorname{bracket}\left(\hat{a}_{n}, \hat{b}_{n}, 0.5\left(\hat{a}_{n}+\hat{b}_{n}\right), a_{n+1}, b_{n+1}\right)$.

\section{Algorithm B2}

set $a_{1}=a, b_{1}=b$, for $n=1,2, \ldots$ do:

B2.1 $c_{n}=a_{n}-f\left[a_{n}, b_{n}\right]^{-1} f\left(a_{n}\right)$;

B2.2 call bracket $\left(a_{n}, b_{n}, c_{n}, \tilde{a}_{n}, \tilde{b}_{n}\right)$;

B2.3 $\tilde{c}_{n}=$ the unique zero of $P\left(a_{n}, b_{n}, c_{n}\right)(x)$ in $\left[\tilde{a}_{n}, \tilde{b}_{n}\right]$;

B2.4 call bracket $\left(\tilde{a}_{n}, \tilde{b}_{n}, \tilde{c}_{n}, \bar{a}_{n}, \bar{b}_{n}\right)$;

B2.5-B2.9: same as B1.3-B1.7.

\section{Algorithm B3}

set $a_{1}=a, b_{1}=b$, for $n=1,2, \ldots$ do:

B3.1 $c_{n}=0.5\left(a_{n}+b_{n}\right)$;

B3.2-B3.6: same as B2.2-B2.6;

B3.7 call bracket $\left(\bar{a}_{n}, \bar{b}_{n}, \bar{c}_{n}, a_{n+1}, b_{n+1}\right)$.

\section{ACKNOWLEDGMENT}

We thank Dr. D. Le for kindly sending us the Fortran code of his Algorithm LZ4, and an anonymous referee whose comments led to an improved presentation of our results.

\section{BIBLIOGRAPHY}

1. G. Alefeld and F. A. Potra, On two higher order enclosing methods of J. W. Schmidt, Z. Angew. Math. Mech. 68 (1988), 331-337.

2. __ Some efficient methods for enclosing simple zeroes of nonlinear equations, BIT 32 (1992), 334-344. 
3. Ned Anderson and Ake Björck, $A$ new high order method of regula falsi type for computing a root of an equation, BIT 13 (1973), 253-264.

4. Ken Atkinson, An introduction to numerical analysis, Wiley, New York, 1989.

5. R. P. Brent, Algorithms for minimization without derivatives, Prentice-Hall, Englewood Cliffs, NJ, 1972.

6. T. J. Dekker, Finding a zero by means of successive linear interpolation, Constructive Aspects of the Fundamental Theorem of Algebra (B. Dejon and P. Henrici, eds.), WileyInterscience, London, 1969, pp. 37-48.

7. R. F. King, Methods without secant steps for finding a bracketed root, Computing 17 (1976), 49-57.

8. D. Le, An efficient derivative-free method for solving nonlinear equations, ACM Trans. Math. Software 11 (1985), 250-262.

9. __ Three new rapidly convergent algorithms for finding a zero of a function, SIAM J. Sci. Statist. Comput. 16 (1985), 193-208.

10. A. M. Ostrowski, Solution of equations in Banach spaces, Academic Press, New York, 1973.

11. F. A. Potra, On Q-order and R-order of convergence, J. Optim. Theory Appl. 63 (1989), 415-431.

12. J. W. Schmidt, Eingrenzung der Lösungen nichtlinearer Gleichungen mit höherer Konvergenzgeschwindigkeit, Computing 8 (1971), 208-215.

Institut für ANgewandte Mathematik, Universität KarlsRuhe, Kaiserstrasse 12, W7500 KARLSRUHE, GERMANY

Department of Mathematics, University of lowa, Iowa City, Iowa 52242

Department of Mathematics and Computer Science, Bloomsburg University, BloomsBurg, PenNSYlVania 17815 